\title{
Dielectronic recombination for boronlike ions
}

\author{
M. H. Chen and K. J. Reed \\ Lawrence Livermore National Laboratory, University of California, P.O. Box 808, Livermore, California 94550 \\ D. S. Guo \\ Department of Physics, Southern University and A\&M College, Baton Rogue, Louisiana 70813 \\ D. W. Savin \\ Columbia Astrophysics Laboratory, Columbia University, New York, New York 10027
}

(Received 5 June 1998)

\begin{abstract}
We have calculated the total dielectronic recombination (DR) coefficients for the ${ }^{2} P_{1 / 2}$ and ${ }^{2} P_{3 / 2}$ states in B-like $\mathrm{Ti}^{17+}, \mathrm{Fe}^{21+}$, and $\mathrm{Mo}^{37+}$ ions for electron temperatures $0.1 \leqslant T \leqslant 10000 \mathrm{eV}$. The calculations are carried out using the multiconfiguration Dirac-Fock method in intermediate coupling with a configuration interaction. We find that accurate Coster-Kronig energies are critical for a successful determination of lowtemperature DR coefficients. We also find that the DR involving fine-structure excitations can be as important as the $2 s-2 p$ excitation channels in the low-temperature regime for some ions. [S1050-2947(98)02612-2]

PACS number(s): $34.80 . \mathrm{Kw}$
\end{abstract}

\section{INTRODUCTION}

Dielectronic recombination (DR) is one of the most important recombination processes in high-temperature plasmas [1-3]. Knowledge of DR rate coefficients is essential for calculations of the ionization balance and kinetics of a plasma $[4,5]$. In early modeling of high-temperature plasmas, the DR rate coefficients were often obtained using the semiempirical Burgess-Merts formula [2,6]. Recently, many $a b$ initio calculations have been performed to determine DR cross sections and rate coefficients [7]. For the boron isoelectronic sequence, DR rate coefficients for a few ions have been calculated, mainly using nonrelativistic wave functions in $L S$ or intermediate coupling [8-10]. Badnell's work on $\mathrm{Fe}^{21+}[10]$ is the only calculation for total rate coefficients. For intrashell DR resonances $(\Delta n=0)$, the existing DR calculations include only the $2 s-2 p$ excitation channel. The excitation between fine-structure levels $2 p_{1 / 2}$ and $2 p_{3 / 2}$ is completely ignored. Recently, the DR channels involving $2 p$ fine-structure excitations have been observed for F-like iron and they are found to be the dominant DR channels in the low-temperature regime [11].

In this work we report a relativistic calculation for the DR rate coefficients in $\mathrm{Ti}^{17+}, \mathrm{Fe}^{21+}$, and $\mathrm{Mo}^{37+}$ including $2 s-2 p, 2 p_{1 / 2}-2 p_{3 / 2}, 2 \ell-3 \ell^{\prime}$, and $1 s-2 p$ excitations. These calculations are carried out using the multiconfiguration Dirac-Fock (MCDF) method in intermediate coupling with a configuration interaction from the same complex [12].

\section{THEORETICAL CALCULATION}

In the isolated resonance approximation, the total DR rate coefficient for an initial state $i$ after averaging over the Maxwellian distribution of the plasma electrons is given by [1]

$$
\begin{aligned}
\alpha_{D R}(i)= & \frac{1}{2 g_{i}}\left(\frac{4 \pi R}{k T}\right)^{3 / 2} a_{0}^{3} \sum_{d, f} \exp \left(-E_{d} / k T\right) \\
& \times g_{d} A_{A}(d \rightarrow i) A_{r}(d \rightarrow f) /\left[\Gamma_{r}(d)+\Gamma_{A}(d)\right] .
\end{aligned}
$$

Here the sums on $d$ and $f$ are over the intermediate autoionizing states $d$ and the stabilized final states $f$, respectively; $g_{d}$ and $g_{i}$ are the statistical weight factors; $R$ is the Rydberg energy and $a_{0}$ is the Bohr radius; $A_{A}(d \rightarrow i)$ is the Auger rate and $A_{r}(d \rightarrow f)$ is the radiative rate; $E_{d}$ is the Auger energy; $k$ is the Boltzmann constant; $T$ is the electron temperature; and $\Gamma_{r}(d)$ and $\Gamma_{A}(d)$ are the total radiative and Auger rates for state $d$, respectively.

From perturbation theory, the Auger transition rate in a frozen-orbital approximation is $[14,15]$.

$$
A_{A}(d \rightarrow i)=\frac{2 \pi}{\hbar}\left|\left\langle\psi_{i}\left|\sum_{\alpha<\beta} V_{\alpha, \beta}\right| \psi_{d}\right\rangle\right|^{2} \rho(\varepsilon),
$$

where $\rho(\varepsilon)$ is the density of final states and the two-electron operator $V_{\alpha \beta}$ is the Coulomb operator. The spontaneous electric-dipole radiative transition rate is given by $[14,16]$

$$
A_{r}(d \rightarrow f)=\frac{2 \pi}{3\left(2 J_{d}+1\right)}\left|\left\langle f\left\|T_{1}\right\| d\right\rangle\right|^{2},
$$

where $\left\langle f\left\|T_{1}\right\| d\right\rangle$ is the electric-dipole reduced matrix element $[14,16]$.

In the present work, the transition matrix elements in Eqs. (2) and (3) for each autoionizing state are evaluated in the framework of the MCDF model. The calculations are performed in the average-level scheme [13] and in intermediate coupling including a configuration interaction from all states having the same principal quantum numbers. In the calculations of energies, we include the contributions not only from the Coulomb interaction but also from the Breit interaction and from quantum electrodynamic corrections. In addition, the effects of orbital relaxation on transition energies are also taken into account by performing separate self-consistent field calculations for the initial and final states (i.e., the $\Delta \mathrm{SCF}$ procedure). In the calculations of matrix elements, however, we employ only the initial-state wave functions.

DR from a state of a B-like ion to a state of a C-like ion with excitations from the $n=2$ shell can be represented by 
TABLE I. Dielectronic recombination rate coefficients $\left(\mathrm{cm}^{3} \mathrm{~s}^{-1}\right)$ from $2 s-2 p, 2 p_{1 / 2}-2 p_{3 / 2}$, and $\Delta n \neq 0$ excitations for Ti ${ }^{17+}$. Numbers in square brackets indicate multiplicative powers of 10 .

\begin{tabular}{|c|c|c|c|c|c|c|c|}
\hline \multirow[b]{2}{*}{$T(\mathrm{eV})$} & \multicolumn{4}{|c|}{${ }^{2} P_{1 / 2}$} & \multicolumn{3}{|c|}{${ }^{2} P_{3 / 2}$} \\
\hline & $2 s-2 p$ & $2 p_{1 / 2}-2 p_{3 / 2}$ & $\Delta n \neq 0$ & Total & $2 s-2 p$ & $\Delta n \neq 0$ & Total \\
\hline 0.1 & $6.484[-13]$ & $5.922[-13]$ & & $1.241[-12]$ & $5.528[-10]$ & & $5.528[-10]$ \\
\hline 0.2 & $1.187[-11]$ & $1.485[-11]$ & & $2.672[-11]$ & $1.531[-09]$ & & $1.531[-09]$ \\
\hline 0.3 & $3.680[-11]$ & $3.693[-11]$ & & $7.373[-11]$ & $1.908[-09]$ & & $1.908[-09]$ \\
\hline 0.4 & $6.839[-11]$ & $5.479[-11]$ & & $1.232[-10]$ & $2.002[-09]$ & & $2.002[-09]$ \\
\hline 0.5 & $1.008[-10]$ & $6.724[-11]$ & & $1.680[-10]$ & $1.968[-09]$ & & $1.968[-09]$ \\
\hline 0.6 & $1.316[-10]$ & $7.557[-11]$ & & $2.072[-10]$ & $1.879[-09]$ & & $1.879[-09]$ \\
\hline 0.7 & $1.597[-10]$ & $8.104[-11]$ & & $2.407[-10]$ & $1.771[-09]$ & & $1.771[-09]$ \\
\hline 0.8 & $1.846[-10]$ & $8.456[-11]$ & & $2.692[-10]$ & $1.660[-09]$ & & $1.660[-09]$ \\
\hline 0.9 & $2.063[-10]$ & $8.673[-11]$ & & $2.930[-10]$ & $1.555[-09]$ & & $1.555[-09]$ \\
\hline 1 & $2.249[-10]$ & $8.794[-11]$ & & $3.128[-10]$ & $1.458[-09]$ & & $1.458[-09]$ \\
\hline 2 & $3.002[-10]$ & $8.082[-11]$ & & $3.810[-10]$ & $8.830[-10]$ & & $8.830[-10]$ \\
\hline 3 & $2.974[-10]$ & $6.714[-11]$ & & $3.645[-10]$ & $6.374[-10]$ & & $6.374[-10]$ \\
\hline 4 & $2.780[-10]$ & $5.543[-11]$ & & $3.334[-10]$ & $5.015[-10]$ & & $5.015[-10]$ \\
\hline 5 & $2.567[-10]$ & $4.627[-11]$ & & $3.030[-10]$ & $4.154[-10]$ & & $4.154[-10]$ \\
\hline 6 & $2.373[-10]$ & $3.918[-11]$ & & $2.765[-10]$ & $3.564[-10]$ & & $3.564[-10]$ \\
\hline 7 & $2.207[-10]$ & $3.365[-11]$ & & $2.544[-10]$ & $3.138[-10]$ & & $3.138[-10]$ \\
\hline 8 & $2.065[-10]$ & $2.925[-11]$ & & $2.358[-10]$ & $2.817[-10]$ & & $2.817[-10]$ \\
\hline 9 & $1.946[-10]$ & $2.572[-11]$ & & $2.203[-10]$ & $2.568[-10]$ & & $2.568[-10]$ \\
\hline 10 & $1.846[-10]$ & $2.282[-11]$ & & $2.074[-10]$ & $2.371[-10]$ & & $2.371[-10]$ \\
\hline 20 & $1.387[-10]$ & $9.655[-12]$ & $2.054[-15]$ & $1.484[-10]$ & $1.551[-10]$ & $2.542[-15]$ & $1.551[-10]$ \\
\hline 30 & $1.223[-10]$ & $5.591[-12]$ & $6.652[-14]$ & $1.280[-10]$ & $1.284[-10]$ & $7.338[-14]$ & $1.285[-10]$ \\
\hline 40 & $1.092[-10]$ & $3.746[-12]$ & $3.556[-13]$ & $1.133[-10]$ & $1.107[-10]$ & $3.727[-13]$ & $1.111[-10]$ \\
\hline 50 & $9.718[-11]$ & $2.732[-12]$ & $9.333[-13]$ & $1.008[-10]$ & $9.658[-11]$ & $9.515[-13]$ & $9.753[-11]$ \\
\hline 60 & $8.652[-11]$ & $2.105[-12]$ & $1.743[-12]$ & $9.037[-11]$ & $8.483[-11]$ & $1.748[-12]$ & $8.658[-11]$ \\
\hline 70 & $7.726[-11]$ & $1.686[-12]$ & $2.710[-12]$ & $8.166[-11]$ & $7.502[-11]$ & $2.692[-12]$ & $7.771[-11]$ \\
\hline 80 & $6.930[-11]$ & $1.388[-12]$ & $3.783[-12]$ & $7.447[-11]$ & $6.682[-11]$ & $3.738[-12]$ & $7.056[-11]$ \\
\hline 90 & $6.246[-11]$ & $1.170[-12]$ & $4.929[-12]$ & $6.856[-11]$ & $5.992[-11]$ & $4.855[-12]$ & $6.478[-11]$ \\
\hline 100 & $5.660[-11]$ & $1.003[-12]$ & $6.122[-12]$ & $6.373[-11]$ & $5.406[-11]$ & $6.019[-12]$ & $6.008[-11]$ \\
\hline 200 & $2.646[-11]$ & $3.615[-13]$ & $1.653[-11]$ & $4.335[-11]$ & $2.481[-11]$ & $1.621[-11]$ & $4.102[-11]$ \\
\hline 300 & $1.587[-11]$ & $1.981[-13]$ & $2.056[-11]$ & $3.663[-11]$ & $1.480[-11]$ & $2.014[-11]$ & $3.494[-11]$ \\
\hline 400 & $1.083[-11]$ & $1.290[-13]$ & $2.074[-11]$ & $3.170[-11]$ & $1.007[-11]$ & $2.029[-11]$ & $3.036[-11]$ \\
\hline 500 & $7.984[-12]$ & $9.252[-14]$ & $1.947[-11]$ & $2.755[-11]$ & $7.410[-12]$ & $1.904[-11]$ & $2.645[-11]$ \\
\hline 600 & $6.196[-12]$ & $7.048[-14]$ & $1.782[-11]$ & $2.409[-11]$ & $5.744[-12]$ & $1.740[-11]$ & $2.314[-11]$ \\
\hline 700 & $4.988[-12]$ & $5.598[-14]$ & $1.617[-11]$ & $2.121[-11]$ & $4.620[-12]$ & $1.579[-11]$ & $2.041[-11]$ \\
\hline 800 & $4.127[-12]$ & $4.584[-14]$ & $1.465[-11]$ & $1.882[-11]$ & $3.820[-12]$ & $1.430[-11]$ & $1.812[-11]$ \\
\hline 900 & $3.488[-12]$ & $3.845[-14]$ & $1.330[-11]$ & $1.683[-11]$ & $3.227[-12]$ & $1.298[-11]$ & $1.621[-11]$ \\
\hline 1000 & $2.997[-12]$ & $3.284[-14]$ & $1.211[-11]$ & $1.514[-11]$ & $2.773[-12]$ & $1.183[-11]$ & $1.460[-11]$ \\
\hline 1400 & $1.841[-12]$ & $1.985[-14]$ & $8.682[-12]$ & $1.054[-11]$ & $1.702[-12]$ & $8.485[-12]$ & $1.019[-11]$ \\
\hline 2000 & $1.092[-12]$ & $1.163[-14]$ & $5.833[-12]$ & $6.937[-12]$ & $1.009[-12]$ & $5.715[-12]$ & $6.724[-12]$ \\
\hline 2400 & $8.354[-13]$ & $8.851[-15]$ & $4.698[-12]$ & $5.542[-12]$ & $7.711[-13]$ & $4.610[-12]$ & $5.381[-12]$ \\
\hline 3000 & $6.007[-13]$ & $6.336[-15]$ & $3.567[-12]$ & $4.174[-12]$ & $5.544[-13]$ & $3.509[-12]$ & $4.063[-12]$ \\
\hline 3400 & $4.991[-13]$ & $5.252[-15]$ & $3.045[-12]$ & $3.549[-12]$ & $4.606[-13]$ & $2.999[-12]$ & $3.460[-12]$ \\
\hline 4000 & $3.922[-13]$ & $4.116[-15]$ & $2.468[-12]$ & $2.864[-12]$ & $3.618[-13]$ & $2.435[-12]$ & $2.797[-12]$ \\
\hline 4400 & $3.404[-13]$ & $3.568[-15]$ & $2.178[-12]$ & $2.522[-12]$ & $3.141[-13]$ & $2.151[-12]$ & $2.465[-12]$ \\
\hline 5000 & $2.815[-13]$ & $2.946[-15]$ & $1.838[-12]$ & $2.122[-12]$ & $2.597[-13]$ & $1.818[-12]$ & $2.078[-12]$ \\
\hline
\end{tabular}

$$
\begin{aligned}
2 s^{2} 2 p+e \rightarrow 2 s 2 p^{2} n \ell \rightarrow & \left(2 s^{2} 2 p n \ell+2 s^{2} 2 p^{2}+2 s 2 p^{3}\right. \\
& \left.+2 s 2 p^{2} n^{\prime} \ell^{\prime}\right)+h \nu
\end{aligned}
$$

$$
\begin{aligned}
2 s^{2} 2 p+e & \rightarrow\left(2 s 2 p n \ell n^{\prime} \ell^{\prime}+2 s^{2} n \ell n^{\prime} \ell^{\prime}, n, n^{\prime} \geqslant 3\right) \\
& \rightarrow\left(2 s^{2} 2 p n^{\prime \prime} \ell^{\prime \prime}+2 s 2 p^{2} n^{\prime \prime} \ell^{\prime \prime}\right)+h \nu ;
\end{aligned}
$$

for the $1 s^{2} 2 s^{2} 2 p^{2} P_{1 / 2}$ state we also include contributions from the fine-structure excitations

$$
\begin{aligned}
2 s^{2} 2 p_{1 / 2}+e & \rightarrow 2 s^{2} 2 p_{3 / 2} n \ell \\
& \rightarrow\left(2 s^{2} 2 p^{2}+2 s^{2} 2 p_{3 / 2} n^{\prime} \ell^{\prime}\right)+h \nu .
\end{aligned}
$$


TABLE II. Dielectronic recombination rate coefficients $\left(\mathrm{cm}^{3} \mathrm{~s}^{-1}\right)$ from $2 s-2 p, 2 p_{1 / 2}-2 p_{3 / 2}$, and $\Delta n \neq 0$ excitations for Fe $\mathrm{e}^{21+}$. Numbers in square brackets denote multiplicative powers of 10 .

\begin{tabular}{|c|c|c|c|c|c|c|c|}
\hline \multirow[b]{2}{*}{$T(\mathrm{eV})$} & \multicolumn{4}{|c|}{${ }^{2} P_{1 / 2}$} & \multicolumn{3}{|c|}{${ }^{2} P_{3 / 2}$} \\
\hline & $2 s-2 p$ & $2 p_{1 / 2}-2 p_{3 / 2}$ & $\Delta n \neq 0$ & Total & $2 s-2 p$ & $\Delta n \neq 0$ & Total \\
\hline 0.1 & $2.873[-09]$ & $1.139[-14]$ & & $2.873[-09]$ & $1.052[-09]$ & & $1.052[-09]$ \\
\hline 0.2 & $5.466[-09]$ & $3.278[-12]$ & & $5.469[-09]$ & $1.064[-09]$ & & $1.064[-09]$ \\
\hline 0.3 & $5.927[-09]$ & $1.712[-11]$ & & $5.944[-09]$ & $8.259[-10]$ & & $8.259[-10]$ \\
\hline 0.4 & $5.803[-09]$ & $3.527[-11]$ & & $5.838[-09]$ & $6.459[-10]$ & & $6.459[-10]$ \\
\hline 0.5 & $5.499[-09]$ & $5.162[-11]$ & & $5.551[-09]$ & $5.226[-10]$ & & $5.226[-10]$ \\
\hline 0.6 & $5.141[-09]$ & $6.452[-11]$ & & $5.206[-09]$ & $4.384[-10]$ & & $4.384[-10]$ \\
\hline 0.7 & $4.779[-09]$ & $7.418[-11]$ & & $4.853[-09]$ & $3.805[-10]$ & & $3.805[-10]$ \\
\hline 0.8 & $4.433[-09]$ & $8.127[-11]$ & & $4.514[-09]$ & $3.411[-10]$ & & $3.411[-10]$ \\
\hline 0.9 & $4.113[-09]$ & $8.639[-11]$ & & $4.199[-09]$ & $3.146[-10]$ & & $3.146[-10]$ \\
\hline 1 & $3.821[-09]$ & $9.005[-11]$ & & $3.911[-09]$ & $2.972[-10]$ & & $2.972[-10]$ \\
\hline 2 & $2.059[-09]$ & $9.504[-11]$ & & $2.154[-09]$ & $2.820[-10]$ & & $2.820[-10]$ \\
\hline 3 & $1.336[-09]$ & $8.724[-11]$ & & $1.423[-09]$ & $2.886[-10]$ & & $2.886[-10]$ \\
\hline 4 & $9.759[-10]$ & $7.869[-11]$ & & $1.055[-09]$ & $2.838[-10]$ & & $2.838[-10]$ \\
\hline 5 & $7.685[-10]$ & $7.075[-11]$ & & $8.393[-10]$ & $2.745[-10]$ & & $2.745[-10]$ \\
\hline 6 & $6.362[-10]$ & $6.367[-11]$ & & $6.999[-10]$ & $2.641[-10]$ & & $2.641[-10]$ \\
\hline 7 & $5.454[-10]$ & $5.747[-11]$ & & $6.029[-10]$ & $2.535[-10]$ & & $2.535[-10]$ \\
\hline 8 & $4.794[-10]$ & $5.207[-11]$ & & $5.315[-10]$ & $2.436[-10]$ & & $2.436[-10]$ \\
\hline 9 & $4.294[-10]$ & $4.738[-11]$ & & $4.768[-10]$ & $2.344[-10]$ & & $2.344[-10]$ \\
\hline 10 & $3.903[-10]$ & $4.330[-11]$ & & $4.336[-10]$ & $2.259[-10]$ & & $2.259[-10]$ \\
\hline 20 & $2.266[-10]$ & $2.128[-11]$ & & $2.479[-10]$ & $1.767[-10]$ & & $1.767[-10]$ \\
\hline 30 & $1.785[-10]$ & $1.305[-11]$ & $6.023[-15]$ & $1.916[-10]$ & $1.565[-10]$ & $8.169[-15]$ & $1.565[-10]$ \\
\hline 40 & $1.530[-10]$ & $9.006[-12]$ & $6.734[-14]$ & $1.621[-10]$ & $1.422[-10]$ & $8.209[-14]$ & $1.423[-10]$ \\
\hline 50 & $1.347[-10]$ & $6.688[-12]$ & $2.742[-13]$ & $1.417[-10]$ & $1.292[-10]$ & $3.149[-13]$ & $1.295[-10]$ \\
\hline 60 & $1.199[-10]$ & $5.217[-12]$ & $6.760[-13]$ & $1.258[-10]$ & $1.173[-10]$ & $7.472[-13]$ & $1.180[-10]$ \\
\hline 70 & $1.075[-10]$ & $4.215[-12]$ & $1.259[-12]$ & $1.130[-10]$ & $1.065[-10]$ & $1.355[-12]$ & $1.079[-10]$ \\
\hline 80 & $9.699[-11]$ & $3.497[-12]$ & $1.977[-12]$ & $1.025[-10]$ & $9.687[-11]$ & $2.090[-12]$ & $9.896[-11]$ \\
\hline 90 & $8.793[-11]$ & $2.962[-12]$ & $2.784[-12]$ & $9.368[-11]$ & $8.837[-11]$ & $2.904[-12]$ & $9.127[-11]$ \\
\hline 100 & $8.012[-11]$ & $2.551[-12]$ & $3.646[-12]$ & $8.632[-11]$ & $8.090[-11]$ & $3.764[-12]$ & $8.466[-11]$ \\
\hline 200 & $3.879[-11]$ & $9.372[-13]$ & $1.235[-11]$ & $5.208[-11]$ & $3.988[-11]$ & $1.236[-11]$ & $5.224[-11]$ \\
\hline 300 & $2.363[-11]$ & $5.167[-13]$ & $1.813[-11]$ & $4.228[-11]$ & $2.441[-11]$ & $1.809[-11]$ & $4.250[-11]$ \\
\hline 400 & $1.626[-11]$ & $3.378[-13]$ & $2.059[-11]$ & $3.719[-11]$ & $1.683[-11]$ & $2.051[-11]$ & $3.734[-11]$ \\
\hline 500 & $1.205[-11]$ & $2.427[-13]$ & $2.101[-11]$ & $3.330[-11]$ & $1.249[-11]$ & $2.091[-11]$ & $3.340[-11]$ \\
\hline 600 & $9.386[-12]$ & $1.851[-13]$ & $2.041[-11]$ & $2.998[-11]$ & $9.733[-12]$ & $2.030[-11]$ & $3.003[-11]$ \\
\hline 700 & $7.575[-12]$ & $1.471[-13]$ & $1.936[-11]$ & $2.708[-11]$ & $7.860[-12]$ & $1.926[-11]$ & $2.712[-11]$ \\
\hline 800 & $6.280[-12]$ & $1.206[-13]$ & $1.816[-11]$ & $2.456[-11]$ & $6.518[-12]$ & $1.804[-11]$ & $2.456[-11]$ \\
\hline 900 & $5.315[-12]$ & $1.012[-13]$ & $1.695[-11]$ & $2.237[-11]$ & $5.519[-12]$ & $1.683[-11]$ & $2.235[-11]$ \\
\hline 1000 & $4.575[-12]$ & $8.647[-14]$ & $1.577[-11]$ & $2.043[-11]$ & $4.751[-12]$ & $1.567[-11]$ & $2.042[-11]$ \\
\hline 1400 & $2.819[-12]$ & $5.232[-14]$ & $1.196[-11]$ & $1.483[-11]$ & $2.930[-12]$ & $1.187[-11]$ & $1.480[-11]$ \\
\hline 2000 & $1.676[-12]$ & $3.070[-14]$ & $8.383[-12]$ & $1.009[-11]$ & $1.743[-12]$ & $8.325[-12]$ & $1.007[-11]$ \\
\hline 2400 & $1.283[-12]$ & $2.336[-14]$ & $6.862[-12]$ & $8.168[-12]$ & $1.335[-12]$ & $6.820[-12]$ & $8.155[-12]$ \\
\hline 3000 & $9.237[-13]$ & $1.673[-14]$ & $5.302[-12]$ & $6.242[-12]$ & $9.607[-13]$ & $5.276[-12]$ & $6.237[-12]$ \\
\hline 3400 & $7.678[-13]$ & $1.387[-14]$ & $4.563[-12]$ & $5.345[-12]$ & $7.986[-13]$ & $4.544[-12]$ & $5.343[-12]$ \\
\hline 4000 & $6.036[-13]$ & $1.088[-14]$ & $3.736[-12]$ & $4.350[-12]$ & $6.279[-13]$ & $3.724[-12]$ & $4.352[-12]$ \\
\hline 4400 & $5.241[-13]$ & $9.426[-15]$ & $3.314[-12]$ & $3.848[-12]$ & $5.452[-13]$ & $3.307[-12]$ & $3.852[-12]$ \\
\hline 5000 & $4.335[-13]$ & $7.783[-15]$ & $2.815[-12]$ & $3.256[-12]$ & $4.510[-13]$ & $2.811[-12]$ & $3.262[-12]$ \\
\hline
\end{tabular}

$1 s^{2} 2 s^{2} 2 p+e \rightarrow 1 s 2 s^{2} 2 p n \ell n^{\prime} \ell^{\prime}$

$$
\rightarrow\left(1 s^{2} 2 s^{2} n \ell n^{\prime} \ell^{\prime}+1 s^{2} 2 s^{2} 2 p n^{\prime \prime} \ell^{\prime \prime}\right)+h \nu \text {. }
$$

For the $\Delta n=0$ transitions with $2 s-2 p$ [Eq. (4)] and $2 p_{1 / 2}-2 p_{3 / 2}$ [Eq. (6)] excitations, explicit calculations are carried out for intermediate states $n \leqslant 32$ and $\ell \leqslant 12$. For the $2 \ell-3 \ell^{\prime}$ excitations [Eq. (5)], detailed calculations are performed for autoionizing states $n=3, \ell \leqslant 2,3 \leqslant n^{\prime} \leqslant 10$, and $\ell^{\prime} \leqslant 6$. For $K$-shell excitation, we include only intermediate states with $n=2, n^{\prime} \leqslant 8$, and $\ell^{\prime} \leqslant 5$. All the possible electricdipole radiative decays to the stabilized bound states and all Auger channels are included in the computation of the radia- 
TABLE III. Dielectronic recombination rate coefficients $\left(\mathrm{cm}^{3} \mathrm{~s}^{-1}\right)$ from $2 s-2 p, 2 p_{1 / 2}-2 p 3 / 2$, and $\Delta n \neq 0$ excitations for Mo ${ }^{37+}$. Numbers in square brackets denote multliplicative powers of 10.

\begin{tabular}{|c|c|c|c|c|c|c|c|}
\hline \multirow[b]{2}{*}{$T(\mathrm{eV})$} & \multicolumn{4}{|c|}{${ }^{2} P_{1 / 2}$} & \multicolumn{3}{|c|}{${ }^{2} P_{3 / 2}$} \\
\hline & $2 s-2 p$ & $2 p_{1 / 2}-2 p_{3 / 2}$ & $\Delta n \neq 0$ & Total & $2 s-2 p$ & $\Delta n \neq 0$ & Total \\
\hline 0.1 & $9.735[-10]$ & & & $9.735[-10]$ & $3.608[-15]$ & & $3.608[-15]$ \\
\hline 0.2 & $2.089[-09]$ & & & $2.089[-09]$ & $9.835[-13]$ & & $9.835[-13]$ \\
\hline 0.3 & $2.350[-09]$ & & & $2.350[-09]$ & $4.911[-12]$ & & $4.911[-12]$ \\
\hline 0.4 & $2.257[-09]$ & & & $2.257[-09]$ & $9.707[-12]$ & & $9.707[-12]$ \\
\hline 0.5 & $2.063[-09]$ & $1.429[-17]$ & & $2.063[-09]$ & $1.382[-11]$ & & $1.382[-11]$ \\
\hline 0.6 & $1.857[-09]$ & $2.903[-16]$ & & $1.857[-09]$ & $1.744[-11]$ & & $1.744[-11]$ \\
\hline 0.7 & $1.667[-09]$ & $2.479[-15]$ & & $1.667[-09]$ & $2.131[-11]$ & & $2.131[-11]$ \\
\hline 0.8 & $1.499[-09]$ & $1.223[-14]$ & & $1.499[-09]$ & $2.608[-11]$ & & $2.608[-11]$ \\
\hline 0.9 & $1.353[-09]$ & $4.183[-14]$ & & $1.353[-09]$ & $3.202[-11]$ & & $3.202[-11]$ \\
\hline 1 & $1.227[-09]$ & $1.106[-13]$ & & $1.227[-09]$ & $3.911[-11]$ & & $3.911[-11]$ \\
\hline 2 & $5.945[-10]$ & $6.650[-12]$ & & $6.011[-10]$ & $1.233[-10]$ & & $1.233[-10]$ \\
\hline 3 & $4.055[-10]$ & $2.048[-11]$ & & $4.260[-10]$ & $1.694[-10]$ & & $1.694[-10]$ \\
\hline 4 & $3.384[-10]$ & $3.210[-11]$ & & $3.705[-10]$ & $1.866[-10]$ & & $1.866[-10]$ \\
\hline 5 & $3.127[-10]$ & $3.961[-11]$ & & $3.523[-10]$ & $1.930[-10]$ & & $1.930[-10]$ \\
\hline 6 & $3.035[-10]$ & $4.401[-11]$ & & $3.475[-10]$ & $1.965[-10]$ & & $1.965[-10]$ \\
\hline 7 & $3.016[-10]$ & $4.643[-11]$ & & $3.480[-10]$ & $1.995[-10]$ & & $1.995[-10]$ \\
\hline 8 & $3.026[-10]$ & $4.762[-11]$ & & $3.502[-10]$ & $2.032[-10]$ & & $2.032[-10]$ \\
\hline 9 & $3.047[-10]$ & $4.807[-11]$ & & $3.528[-10]$ & $2.073[-10]$ & & $2.073[-10]$ \\
\hline 10 & $3.067[-10]$ & $4.805[-11]$ & & $3.548[-10]$ & $2.115[-10]$ & & $2.115[-10]$ \\
\hline 20 & $2.946[-10]$ & $4.196[-11]$ & & $3.366[-10]$ & $2.361[-10]$ & & $2.361[-10]$ \\
\hline 30 & $2.629[-10]$ & $3.596[-11]$ & & $2.989[-10]$ & $2.326[-10]$ & & $2.326[-10]$ \\
\hline 40 & $2.357[-10]$ & $3.108[-11]$ & & $2.668[-10]$ & $2.209[-10]$ & & $2.209[-10]$ \\
\hline 50 & $2.143[-10]$ & $2.707[-11]$ & & $2.414[-10]$ & $2.081[-10]$ & $1.602[-16]$ & $2.081[-10]$ \\
\hline 60 & $1.973[-10]$ & $2.378[-11]$ & $3.646[-16]$ & $2.211[-10]$ & $1.957[-10]$ & $1.503[-15]$ & $1.957[-10]$ \\
\hline 70 & $1.831[-10]$ & $2.104[-11]$ & $2.308[-15]$ & $2.041[-10]$ & $1.842[-10]$ & $7.474[-15]$ & $1.842[-10]$ \\
\hline 80 & $1.711[-10]$ & $1.876[-11]$ & $9.133[-15]$ & $1.899[-10]$ & $1.734[-10]$ & $2.479[-14]$ & $1.734[-10]$ \\
\hline 90 & $1.605[-10]$ & $1.684[-11]$ & $2.636[-14]$ & $1.774[-10]$ & $1.634[-10]$ & $6.259[-14]$ & $1.635[-10]$ \\
\hline 100 & $1.510[-10]$ & $1.521[-11]$ & $6.095[-14]$ & $1.663[-10]$ & $1.542[-10]$ & $1.303[-13]$ & $1.543[-10]$ \\
\hline 200 & $9.047[-11]$ & $7.049[-12]$ & $2.070[-12]$ & $9.959[-11]$ & $9.236[-11]$ & $2.872[-12]$ & $9.523[-11]$ \\
\hline 300 & $6.098[-11]$ & $4.226[-12]$ & $5.531[-12]$ & $7.074[-11]$ & $6.189[-11]$ & $6.781[-12]$ & $6.867[-11]$ \\
\hline 400 & $4.441[-11]$ & $2.884[-12]$ & $8.469[-12]$ & $5.576[-11]$ & $4.488[-11]$ & $9.828[-12]$ & $5.471[-11]$ \\
\hline 500 & $3.412[-11]$ & $2.126[-12]$ & $1.065[-11]$ & $4.690[-11]$ & $3.440[-11]$ & $1.199[-11]$ & $4.639[-11]$ \\
\hline 600 & $2.725[-11]$ & $1.651[-12]$ & $1.221[-11]$ & $4.111[-11]$ & $2.741[-11]$ & $1.350[-11]$ & $4.091[-11]$ \\
\hline 700 & $2.240[-11]$ & $1.329[-12]$ & $1.329[-11]$ & $3.702[-11]$ & $2.249[-11]$ & $1.451[-11]$ & $3.700[-11]$ \\
\hline 800 & $1.883[-11]$ & $1.099[-12]$ & $1.398[-11]$ & $3.391[-11]$ & $1.889[-11]$ & $1.512[-11]$ & $3.401[-11]$ \\
\hline 900 & $1.611[-11]$ & $9.295[-13]$ & $1.437[-11]$ & $3.141[-11]$ & $1.615[-11]$ & $1.545[-11]$ & $3.160[-11]$ \\
\hline 1000 & $1.399[-11]$ & $7.990[-13]$ & $1.455[-11]$ & $2.934[-11]$ & $1.401[-11]$ & $1.555[-11]$ & $2.956[-11]$ \\
\hline 1400 & $8.821[-12]$ & $4.910[-13]$ & $1.401[-11]$ & $2.332[-11]$ & $8.819[-12]$ & $1.475[-11]$ & $2.357[-11]$ \\
\hline 2000 & $5.341[-12]$ & $2.914[-13]$ & $1.198[-11]$ & $1.761[-11]$ & $5.331[-12]$ & $1.246[-11]$ & $1.779[-11]$ \\
\hline 2400 & $4.116[-12]$ & $2.228[-13]$ & $1.063[-11]$ & $1.497[-11]$ & $4.106[-12]$ & $1.100[-11]$ & $1.511[-11]$ \\
\hline 3000 & $2.983[-12]$ & $1.603[-13]$ & $8.913[-12]$ & $1.206[-11]$ & $2.975[-12]$ & $9.178[-12]$ & $1.215[-11]$ \\
\hline 3400 & $2.488[-12]$ & $1.331[-13]$ & $7.972[-12]$ & $1.059[-11]$ & $2.480[-12]$ & $8.184[-12]$ & $1.066[-11]$ \\
\hline 4000 & $1.964[-12]$ & $1.046[-13]$ & $6.815[-12]$ & $8.884[-12]$ & $1.957[-12]$ & $6.970[-12]$ & $8.927[-12]$ \\
\hline 4400 & $1.708[-12]$ & $9.082[-14]$ & $6.180[-12]$ & $7.979[-12]$ & $1.701[-12]$ & $6.309[-12]$ & $8.010[-12]$ \\
\hline 5000 & $1.416[-12]$ & $7.510[-14]$ & $5.389[-12]$ & $6.880[-12]$ & $1.410[-12]$ & $5.488[-12]$ & $6.898[-12]$ \\
\hline
\end{tabular}

tive branching ratios. The contributions from the high- $n$ Rydberg states $(33 \leqslant n \leqslant 400$ for the $\Delta n=0$ transitions and 11 $\leqslant n^{\prime} \leqslant 200$ for the $\Delta n \neq 0$ transitions with $L$-shell excitation) are taken into account using the $n^{-3}$ scaling to the transition rates. Since the contributions from $K$-shell excitations are small and have a fast convergence, there is no high- $n$ extrapolation for these excitation channels. The onset principal quantum numbers $n_{0}$ at which the $\Delta n=0$ transitions become energetically possible are determined using the MCDF$\Delta \mathrm{SCF}$ procedure for each ion covered in this study.

Since the DR rate coefficients are extremely sensitive to the resonance energies at very low temperatures, we use experimental $2 s-2 p$ excitation energies [17] to calculate the $2 s-2 p n \ell$ DR resonance energies because MCDF energies 


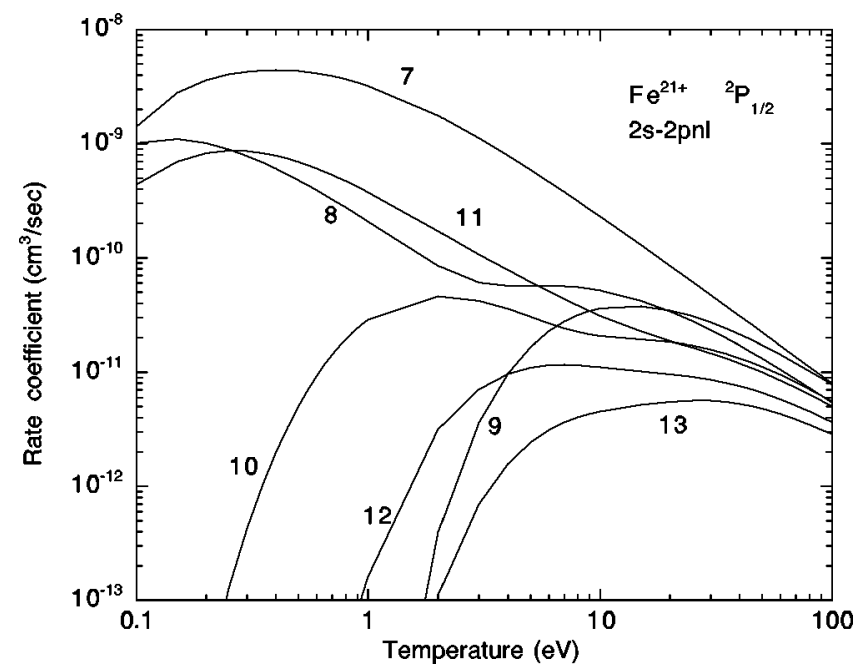

FIG. 1. DR rate coefficients from $2 s-2 p$ excitation for the ${ }^{2} P_{1 / 2}$ state in $\mathrm{Fe}^{21+}$ as functions of electron temperature. The numbers labeling the curves are principal quantum numbers of the Rydberg electrons.

have a few eV uncertainty. For the case of $2 p_{1 / 2}-2 p_{3 / 2} n \ell$ $\mathrm{DR}$, theoretical fine-structure splittings agree with experiment to better than $0.3 \mathrm{eV}$. We then use theoretical energies in these calculations.

\section{RESULTS AND DISCUSSION}

We have calculated the total DR rate coefficients for the $1 s^{2} 2 s^{2} 2 p$ states of B-like ions with $Z=22,26$, and 42 for electron temperatures $0.1 \leqslant T \leqslant 10000 \mathrm{eV}$. The results for $0.1 \leqslant T \leqslant 5000 \mathrm{eV}$ for the $2 s-2 p, 2 p_{1 / 2}-2 p_{3 / 2}$, and $\Delta n \neq 0$ excitations for $\mathrm{Ti}^{17+}, \mathrm{Fe}^{21+}$, and $\mathrm{Mo}^{37+}$ are listed in Tables I, II, and III, respectively. In the $2 s^{2} 2 p+e \rightarrow 2 s 2 p^{2} n \ell$ $\rightarrow 2 s^{2} 2 p_{3 / 2} n \ell$ transitions, the $2 s^{2} 2 p_{3 / 2} n \ell$ states are autoionizing states when $n$ is larger than the onset value $n_{0}$ for the fine-structure transitions. Including the autoionization branching of these autoionizing states reduces the DR coefficients. This cascade effect has been observed recently in

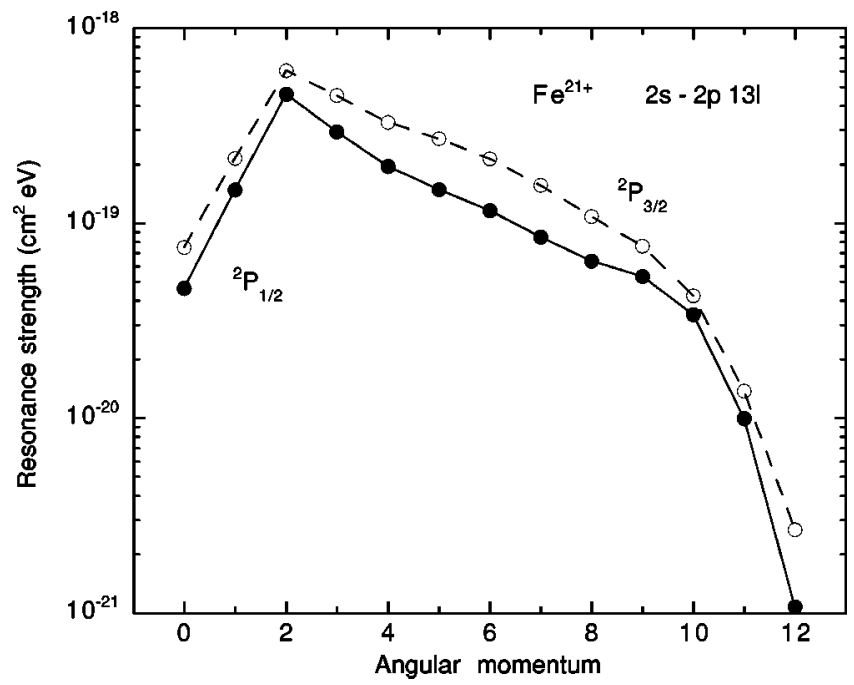

FIG. 2. Resonance strength from $2 s-2 p 13 \ell, \Delta n=0$ transitions in $\mathrm{Fe}^{21+}$ as a function of orbital angular momentum $\ell$.

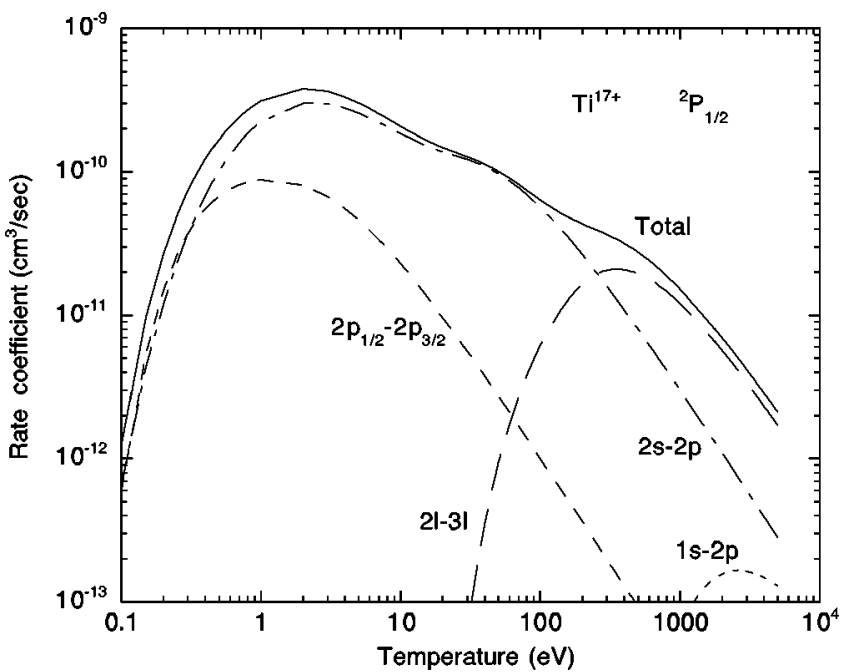

FIG. 3. DR rate coefficients for the ${ }^{2} P_{1 / 2}$ state of $\mathrm{Ti}^{17+}$ as functions of electron temperature. The dash-dotted, short-dashed, longdashed, and dotted curves represent the results from the $2 s-2 p$, $2 p_{1 / 2}-2 p_{3 / 2}, 2 \ell-3 \ell^{\prime}$, and $1 s-2 p$ excitations, respectively. The solid curve displays the total DR rate coefficients.

F-like $\mathrm{Fe}^{17+}[11]$ and $\mathrm{Se}^{25+}$ [18]. Similar cascade effects are also included for the $2 s^{2} 2 p+e \rightarrow 2 s 2 p 3 \ell n \ell \rightarrow 2 s 2 p^{2} n \ell$ transitions when $n$ is larger than a certain value. The results listed in Tables I-III are obtained including this cascade effect.

In Fig. 1 the $n$ dependence of rate coefficients for the ${ }^{2} P_{1 / 2}$ state of $\mathrm{Fe}^{21+}$ with $2 s-2 p$ excitation is displayed. For the $2 s-2 p$ excitation, the order of importance behaves irregularly as a function of $n$ at the low-temperature regime. This is caused by the opening of the low-energy CosterKronig channels as $n$ increases. For $T<10 \mathrm{eV}$, DR rate coefficients are dominated by low-energy resonances. For $n$ $=7$, only the $2 s 2 p^{2}{ }^{2} P$ excitation channel is open with resonance energies ranging from 0.23 to $1.52 \mathrm{eV}$. For $n=8$, $2 s 2 p^{2}{ }^{2} P,{ }^{2} S$, and ${ }^{2} D$ are open with energies from $0.1 \mathrm{eV}$ to $29.6 \mathrm{eV}$. There are 62 transitions with energies less than $10 \mathrm{eV}$. For $n=9$, no new channel is open. The resonance

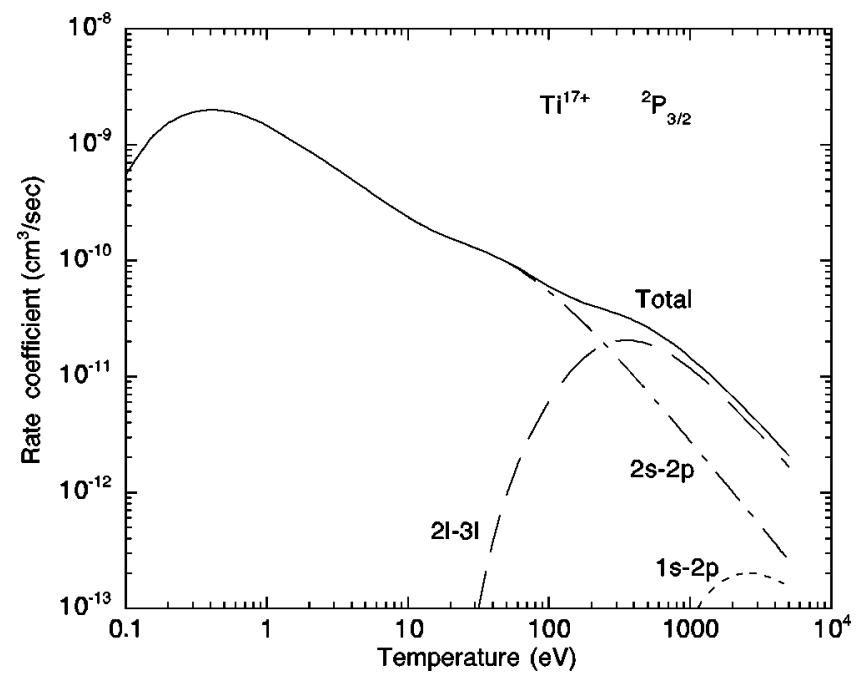

FIG. 4. DR rate coefficients for the ${ }^{2} P_{3 / 2}$ state of $\mathrm{Ti}^{17+}$ as functions of electron temperature. The legend is the same as in Fig. 3. 


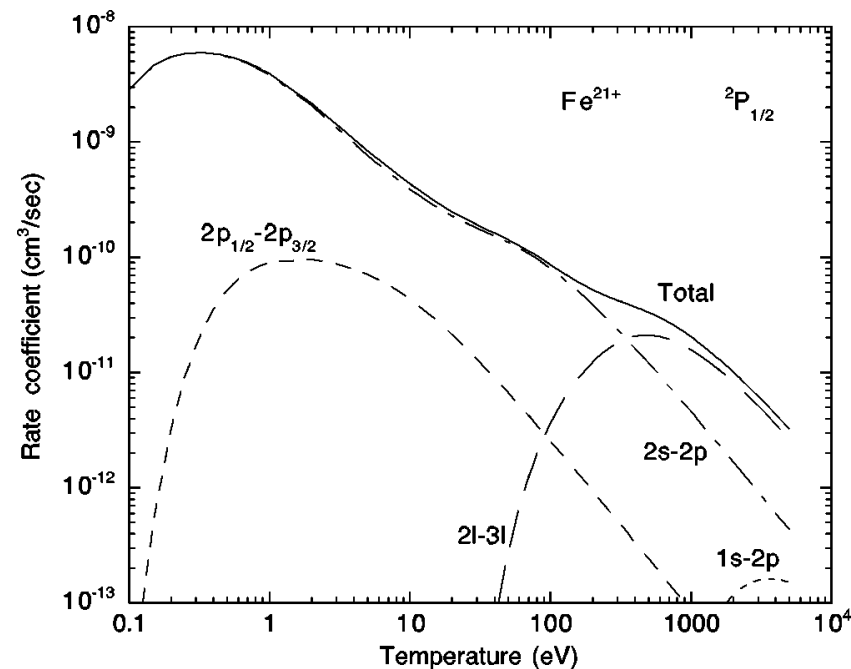

FIG. 5. DR rate coefficients for the ${ }^{2} P_{1 / 2}$ state of $\mathrm{Fe}^{21+}$ as functions of electron temperature. The legend is the same as in Fig. 3.

energies increase to $15-49.3 \mathrm{eV}$. For $n=10$, a new channel ${ }^{4} P_{5 / 2}$ is open with energies in the range $1.9-3.6 \mathrm{eV}$. For $n$ $=11$, the last two excitation channels ${ }^{4} P_{3 / 2}$ and ${ }^{4} P_{1 / 2}$ are open with energies as low as $0.2 \mathrm{eV}$ and there are 98 transitions with energies less than $10 \mathrm{eV}$. For $n=12$, all channels are open with 34 transitions having energies between 7.5 and $10 \mathrm{eV}$. The rise and fall of the number of low energy resonances as a function of $n$ are responsible for the irregular behavior of the $n$ dependence. On the other hand, for the $\Delta n \neq 0$ transitions, contributions decrease with increasing $n$, as expected. The DR rate coefficients from the $\Delta n=0$ transitions at low temperatures are very sensitive to the CosterKronig energy. Hence an accurate determination of the Coster-Kronig energy including the fine-structure splitting is essential for obtaining reliable low-temperature DR rate coefficients. The $\ell$ dependence of the $2 s-2 p 13 \ell$ transitions for $\mathrm{Fe}^{21+}$ is shown in Fig. 2. The DR rate peaks at $\ell=2$ and is still very important even for $\ell$ as large as 8 . For $\ell=12$, the rate has reduced by three orders of magnitude with re-

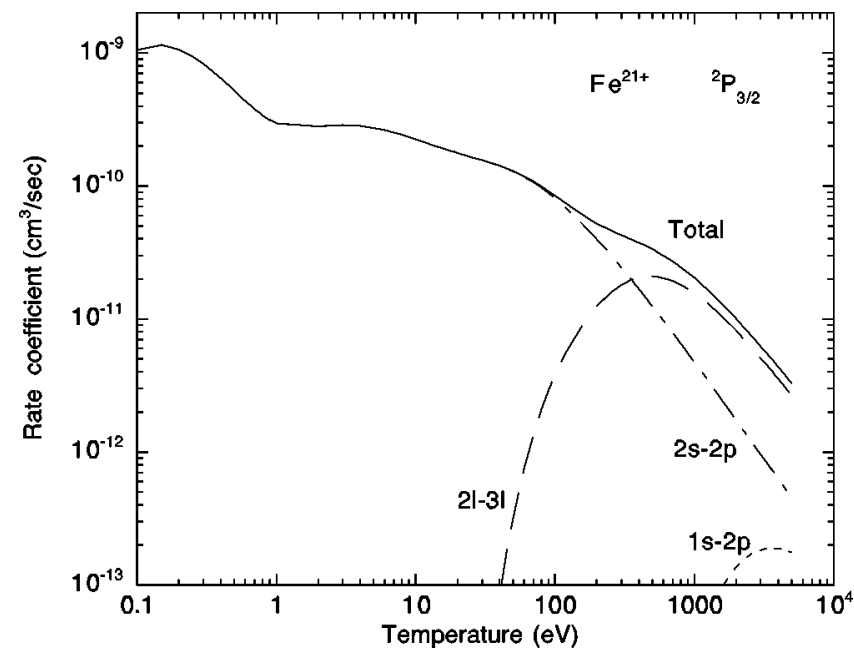

FIG. 6. DR rate coefficients for the ${ }^{2} P_{3 / 2}$ state of $\mathrm{Fe}^{21+}$ as functions of electron temperature. The legend is the same as in Fig. 3.

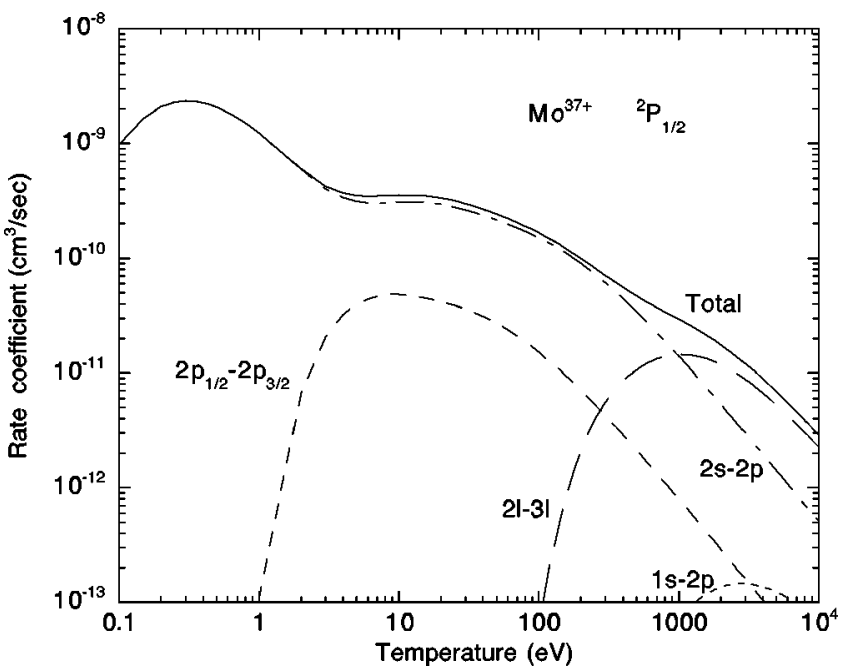

FIG. 7. DR rate coefficients for the ${ }^{2} P_{1 / 2}$ state of $\mathrm{Mo}^{37+}$ as functions of electron temperature. The legend is the same as in Fig. 3.

spect to peak rate. The high- $n$ states with $\ell>12$ in the $\Delta n$ $=0$ transitions contribute only a few percent. They are estimated by fitting the values for $\ell=10,11,12$ to a power law. For the $\Delta n \neq 0$ transitions, no high- $\ell(\ell>6)$ extrapolation is performed.

In this work, the onset $n_{0}$ of the Coster-Kronig channel $2 s-2 p n \ell$ is 7 for all three ions for ${ }^{2} P_{1 / 2}$ state and they are 7 , 8 , and 9 , respectively, for $Z=22,26$, and 42 for the ${ }^{2} P_{3 / 2}$ state. At $n=n_{0}$, some of the states are open for the CosterKronig channels. For the $2 p_{1 / 2}-2 p_{3 / 2} n \ell$ channel, CosterKronig transition becomes energetically possible at $n_{0}=25$, 21 , and 13, respectively, for $Z=22,26$, and 42 .

In Figs. 3-8, DR rate coefficients for the $1 s^{2} 2 s^{2} 2 p{ }^{2} P_{1 / 2,3 / 2}$ states of $\mathrm{Ti}^{17+}, \mathrm{Fe}^{21+}$, and $\mathrm{Mo}^{37+}$ as functions of temperature are displayed. For $T<100 \mathrm{eV}$, the DR rate coefficients are dominated by the $\Delta n=0$ transitions. For the ${ }^{2} P_{1 / 2}$ state, the contributions from the fine-structure excitation [Eq. (6)] to the total DR rate coefficients for $\mathrm{Ti}^{17+}$

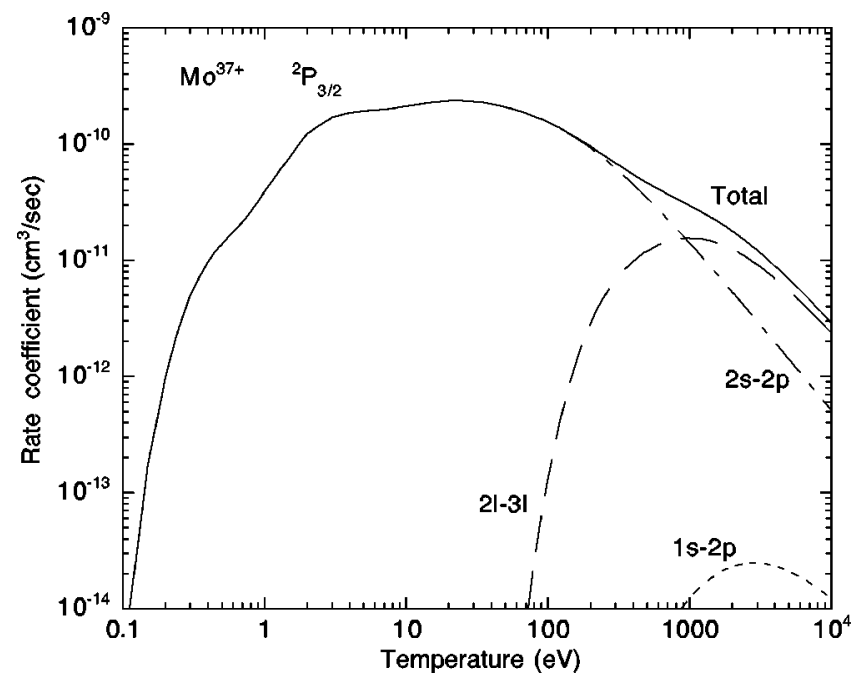

FIG. 8. DR rate coefficients for the ${ }^{2} P_{3 / 2}$ state of $\mathrm{Mo}^{37+}$ as functions of electron temperature. The legend is the same as in Fig. 3. 


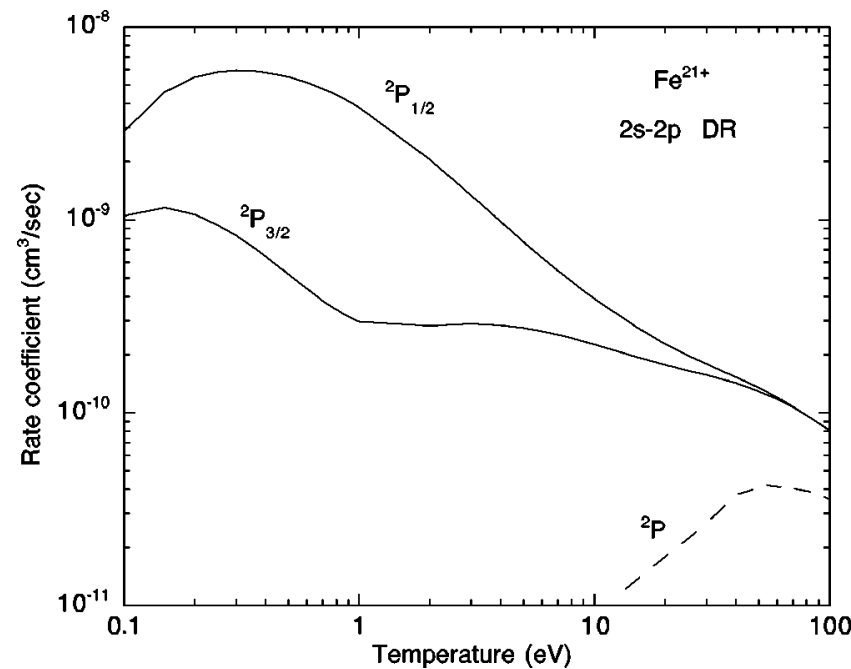

FIG. 9. DR rate coefficients from $\Delta n=0$ transitions with $2 s-2 p$ excitation for $\mathrm{Fe}^{21+}$ as functions of electron temperature. The solid curves display the present results and the dashed curve represents the values from Ramadan and Hahn [8].

are as important as $2 s-2 p$ excitation for $T<1 \mathrm{eV}$. However, for $\mathrm{Fe}^{21+}$ and $\mathrm{Mo}^{37+}$, the fine-structure excitation channel contributes less than $15 \%$.

The relative importance of the fine-structure excitation channel to the $2 s-2 p$ channel depends on the energy level structure of the particular ion. If the atomic structure of an ion happens to provide many low-energy DR resonances with energy less than a few $\mathrm{eV}$ for the $2 s-2 p$ channel, then DR rate coefficients at low temperatures tend to be dominated by this $2 s-2 p$ Coster-Kronig channel such as in $\mathrm{Fe}^{21+}$ and $\mathrm{Mo}^{37+}$.

For higher temperatures, $2 \ell-3 \ell^{\prime}$ excitation becomes the dominant DR channel, while $1 s-2 p$ excitation contributes less than $10 \%$. For $T<50 \mathrm{eV}, \mathrm{DR}$ rates are quite sensitive to resonance energy. The theoretical uncertainties at this energy range are much larger than those at higher temperatures.

The relative importance of radiative recombination (RR) with respect to DR was investigated for $\mathrm{Fe}^{21+}$. For $T \leqslant 0.1$ $\mathrm{eV}, \mathrm{RR}$ [19] is the dominant recombination process. For $0.1 \leqslant T \leqslant 10 \mathrm{eV}$, the ratios between $\mathrm{RR}$ and DR range from 1.39 to 0.22 and the fine-structure transitions contribute less than $8 \%$ to the total recombination rate coefficients.

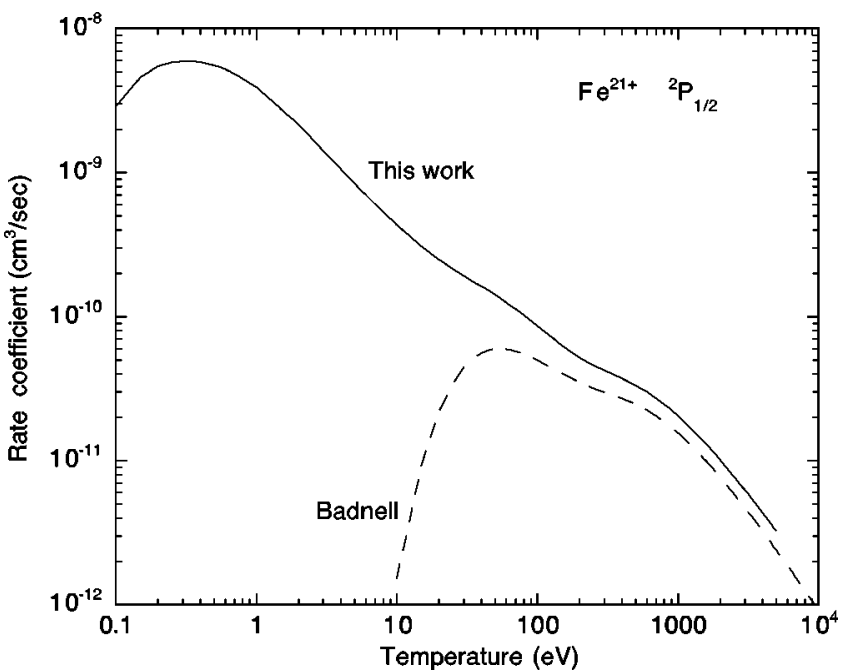

FIG. 10. Total DR rate coefficients for the ${ }^{2} P_{1 / 2}$ state of $\mathrm{Fe}^{21+}$. The solid curve indicates the results from this work and the dashed curve represents the values from Badnell [10].

Experimental measurements of DR rate coefficients for boronlike ions have been carried out by Wang et al. on $\mathrm{Ti}^{17+}$ for $600 \leqslant T \leqslant 1100 \mathrm{eV}[20]$ and on $\mathrm{Fe}^{21+}$ at $T=900 \mathrm{eV}$ [21]. These measurements were obtained using a tokamak plasma with electron densities of $\sim 4 \times 10^{13} \mathrm{~cm}^{-3}$. Our theoretical $\mathrm{Ti}^{17+}$ results in the low-density limit are higher than the measurements and lie 10-40\% outside the experimental uncertainties. Our low-density $\mathrm{Fe}^{21+}$ results are also higher than the experimental values, but lie within the stated factor of 2 uncertainty of the measurement. It is to be expected that our low-density DR rate coefficients are larger than experiment. The effects of electron density [22] and ion density [23] tend to decrease the DR rate coefficients and to bring theory into better agreement with plasma experiment. For example, at ion density of $\sim 2.4 \times 10^{12} \mathrm{~cm}^{-3}$, the continuum lowering due to the Stark broadening limits the Rydberg series to $n \leqslant 23$ for $\mathrm{Ti}^{17+}$. This depression of Rydberg series leads to a $15 \%$ reduction of DR rate coefficient at $T=900 \mathrm{eV}$ for $\mathrm{Ti}^{17+}$. In addition, stepwise electron-impact excitation of Rydberg electron followed by ionization can further reduce the DR rate coefficients.

In Fig. 9, the present DR rate coefficients for the $2 s-2 p$ excitation are compared with the results from nonrelativistic

TABLE IV. $\mathrm{Ti}^{17+}$ dielectronic recombination rate coefficient fit parameters for the various core excitation channels. Numbers in square brackets denote multiplicative powers of 10 .

\begin{tabular}{|c|c|c|c|c|c|c|c|c|c|c|}
\hline \multirow[b]{3}{*}{$i$} & \multicolumn{6}{|c|}{${ }^{2} P_{1 / 2}$} & \multicolumn{4}{|c|}{${ }^{2} P_{3 / 2}$} \\
\hline & \multicolumn{2}{|c|}{$2 s-2 p$} & \multicolumn{2}{|c|}{$2 p_{1 / 2}-2 p_{3 / 2}$} & \multicolumn{2}{|c|}{$\Delta n \neq 0$} & \multicolumn{2}{|c|}{$2 s-2 p$} & \multicolumn{2}{|c|}{$\Delta n \neq 0$} \\
\hline & $c_{i}$ & $E_{i}$ & $c_{i}$ & $E_{i}$ & $c_{i}$ & $E_{i}$ & $c_{i}$ & $E_{i}$ & $c_{i}$ & $E_{i}$ \\
\hline 1 & $8.05[-5]^{\mathrm{a}}$ & $8.40[-1]^{\mathrm{b}}$ & $5.19[-9]$ & $5.73[-3]$ & $9.94[-2]$ & $2.78[2]$ & $9.85[-4]$ & $3.82[-1]$ & $9.25[-2]$ & $2.74[2]$ \\
\hline 2 & $9.36[-4]$ & 1.76 & $1.38[-4]$ & $8.87[-1]$ & $7.40[-1]$ & $6.18[2]$ & $2.31[-3]$ & $9.04[-1]$ & $7.24[-1]$ & $6.13[2]$ \\
\hline 3 & $3.66[-3]$ & 3.76 & $3.67[-4]$ & 2.05 & $1.19[-1]$ & $3.27[3]$ & $1.16[-3]$ & 1.81 & $1.44[-1]$ & $3.36[3]$ \\
\hline 4 & $7.31[-3]$ & 9.54 & $7.81[-4]$ & 4.99 & & & $5.88[-3]$ & 5.56 & & \\
\hline 5 & $2.11[-2]$ & $2.99[1]$ & & & & & $1.71[-2]$ & $2.20[1]$ & & \\
\hline 6 & $9.23[-2]$ & $7.41[1]$ & & & & & $8.69[-2]$ & $6.80[1]$ & & \\
\hline
\end{tabular}

${ }^{\mathrm{a}}$ In units of $\mathrm{cm}^{3} \mathrm{~s}^{-1} \mathrm{~K}^{1.5}$.

${ }^{\mathrm{b}}$ In units of $\mathrm{eV}$. 
TABLE V. $\mathrm{Fe}^{21+}$ dielectronic recombination rate coefficient fit parameters for the various core excitation channels. Numbers in square brackets denote multiplicative powers of 10 .

\begin{tabular}{|c|c|c|c|c|c|c|c|c|c|c|}
\hline \multirow[b]{3}{*}{$i$} & \multicolumn{6}{|c|}{${ }^{2} P_{1 / 2}$} & \multicolumn{4}{|c|}{${ }^{2} P_{3 / 2}$} \\
\hline & \multicolumn{2}{|c|}{$2 s-2 p$} & \multicolumn{2}{|c|}{$2 p_{1 / 2}-2 p_{3 / 2}$} & \multicolumn{2}{|c|}{$\Delta n \neq 0$} & \multicolumn{2}{|c|}{$2 s-2 p$} & \multicolumn{2}{|c|}{$\Delta n \neq 0$} \\
\hline & $c_{i}$ & $E_{i}$ & $c_{i}$ & $E_{i}$ & $c_{i}$ & $E_{i}$ & $c_{i}$ & $E_{i}$ & $c_{i}$ & $E_{i}$ \\
\hline 1 & $2.02[-3]^{\mathrm{a}}$ & $2.93[-1]^{\mathrm{b}}$ & $3.60[-4]$ & 1.39 & $1.93[-1]$ & $3.79[2]$ & $3.40[-4]$ & $2.10[-1]$ & $1.81[-1]$ & $3.69[2]$ \\
\hline 2 & $3.21[-3]$ & $6.28[-1]$ & $8.71[-4]$ & 3.63 & 1.18 & $8.77[2]$ & $2.08[-4]$ & 1.69 & 1.17 & $8.69[2]$ \\
\hline 3 & $6.15[-3]$ & 1.37 & $2.14[-3]$ & 9.92 & $1.94[-1]$ & $4.55[3]$ & $5.88[-3]$ & 4.63 & $2.23[-1]$ & $4.65[3]$ \\
\hline 4 & $5.12[-3]$ & 8.00 & & & & & $1.36[-2]$ & $1.30[1]$ & & \\
\hline 5 & $2.78[-2]$ & $2.29[1]$ & & & & & $3.54[-2]$ & $3.56[1]$ & & \\
\hline 6 & $1.45[-1]$ & $8.46[1]$ & & & & & $1.45[-1]$ & $9.13[1]$ & & \\
\hline
\end{tabular}

${ }^{\mathrm{a}}$ In units of $\mathrm{cm}^{3} \mathrm{~s}^{-1} \mathrm{~K}^{1.5}$.

${ }^{\mathrm{b}}$ In units of $\mathrm{eV}$.

Hartree-Fock calculations in $L S$ coupling by Ramadan and Hahn [8]. Our present results are larger by more than a factor of 2 and peak at much lower temperatures. This is probably due to the differences in the calculated Auger energies. For the $2 s-2 p$ transitions in $\mathrm{Fe}^{21+}$, the onset is $n_{0}=7$ in this work as compared to $n_{0}=12$ in Ref. [8]. If we neglect the contributions from $n=7$ to 11 , then our $2 s-2 p$ DR rates are in fair agreement (better than 30\%) with Ramaden and Hahn and peak near the same temperature.

In Fig. 10, the total DR rate coefficients for the ${ }^{2} P_{1 / 2}$ state of $\mathrm{Fe}^{21+}$ from this work are compared with the results from Badnell [10]. In Badnell's calculations, the contributions from $2 s-2 p$ excitation were computed using Burgess's formula [2]. For $\Delta n \neq 0$ transitions, he employed wave functions from the SUPERSTRUCTURE code in intermediate coupling. For $T>100 \mathrm{eV}$, the two theories agree within $30 \%$. For lower temperatures, they differ by more than an order of magnitude. Most of these differences can be traced to the discrepancies in $2 s-2 p$ excitation and partly to neglect of fine-structure transitions in Badnell's work.

\section{RATES FOR PLASMA MODELING}

We have fit our theoretical DR rate coefficients using the formula [24]

$$
\alpha_{D R}(T)=T^{-3 / 2} \sum_{i} c_{i} e^{-E_{i} / k T}
$$

which has the same functional dependence on $T$ as the Burgess formula [2]. The quantities $c_{i}$ and $E_{i}$ are the strength parameter and the energy parameter, respectively, for the $i$ th fitting function component.

The best fit values for the various DR channels of $\mathrm{Ti}^{17+}$ are listed in Table IV. The fit for the ${ }^{2} P_{1 / 2}$ state $2 s-2 p$ channel is good to better than $4 \%$ for $0.15 \leqslant T \leqslant 10000 \mathrm{eV}$. For $0.1 \leqslant T<0.15 \mathrm{eV}$, the fit is good to better than $30 \%$. The fit for the ${ }^{2} P_{3 / 2}$ state $2 s-2 p$ channel is better than $2 \%$ for $0.1 \leqslant T \leqslant 10000 \mathrm{eV}$. The fit for the $2 p_{1 / 2}-2 p_{3 / 2}$ channel is better than $4 \%$ for $0.1 \leqslant T \leqslant 10000 \mathrm{eV}$. The fits for the $\Delta n$ $\neq 0$ channels are better than $3 \%$ for $60 \leqslant T \leqslant 10000 \mathrm{eV}$. Below $60 \mathrm{eV}$ the fits go to zero faster than the calculations, but this is unimportant as these channels contributes less than $2 \%$ to the total DR rate for $T<60 \mathrm{eV}$.

The best fit values for the various DR channels of $\mathrm{Fe}^{21+}$ are listed in Table V. The fits for the ${ }^{2} P_{1 / 2}$ state and the ${ }^{2} P_{3 / 2}$ state $2 s-2 p$ channels are better than $3 \%$ and $1 \%$, respectively, for $0.1 \leqslant T \leqslant 10000 \mathrm{eV}$. The fit for the $2 p_{1 / 2}-2 p_{3 / 2}$ channel is better than $1 \%$ for $0.3 \leqslant T$ $\leqslant 10000 \mathrm{eV}$ and better than $27 \%$ for $0.1 \leqslant T<0.3 \mathrm{eV}$. The fits for the $\Delta n \neq 0$ channels are better than $1.5 \%$ for $90 \leqslant T$ $\leqslant 10000 \mathrm{eV}$. Below $90 \mathrm{eV}$, the fits go to zero faster than the calculations, but this is unimportant as these channels contribute less than $\sim 3 \%$ to the total DR rate for $T<90 \mathrm{eV}$.

The best fit values for the various DR channels of $\mathrm{Mo}^{37+}$ are listed in Table VI. The fit for the ${ }^{2} P_{1 / 2}$ state $2 s-2 p$

TABLE VI. $\mathrm{Mo}^{37+}$ dielectronic recombination rate coefficient fit parameters for the various core excitation channels. Numbers in square brackets denote multiplicative powers of 10 .

\begin{tabular}{|c|c|c|c|c|c|c|c|c|c|c|}
\hline \multirow[b]{3}{*}{$i$} & \multicolumn{6}{|c|}{${ }^{2} P_{1 / 2}$} & \multicolumn{4}{|c|}{${ }^{2} P_{3 / 2}$} \\
\hline & \multicolumn{2}{|c|}{$2 s-2 p$} & \multicolumn{2}{|c|}{$2 p_{1 / 2}-2 p_{3 / 2}$} & \multicolumn{2}{|c|}{$\Delta n \neq 0$} & \multicolumn{2}{|c|}{$2 s-2 p$} & \multicolumn{2}{|c|}{$\Delta n \neq 0$} \\
\hline & $c_{i}$ & $E_{i}$ & $c_{i}$ & $E_{i}$ & $c_{i}$ & $E_{i}$ & $c_{i}$ & $E_{i}$ & $c_{i}$ & $E_{i}$ \\
\hline 1 & $4.65[-4]^{\mathrm{a}}$ & $2.67[-1]^{b}$ & $4.42[-3]$ & $1.05[1]$ & $7.49[-1]$ & $9.27[2]$ & $9.12[-5]$ & 1.35 & $6.67[-1]$ & $8.40[2]$ \\
\hline 2 & $1.99[-3]$ & $5.77[-1]$ & $8.90[-3]$ & $3.23[1]$ & 1.99 & $2.09[3]$ & $5.65[-3]$ & 5.40 & 1.23 & $1.78[3]$ \\
\hline 3 & $3.10[-4]$ & 1.61 & $1.98[-2]$ & $8.35[1]$ & $8.64[-1]$ & $3.27[3]$ & $9.83[-3]$ & $1.36[1]$ & 1.70 & $2.66[3]$ \\
\hline 4 & $1.50[-2]$ & $1.10[1]$ & & & & & $9.13[-2]$ & $3.62[1]$ & & \\
\hline 5 & $7.80[-2]$ & $2.86[1]$ & & & & & $1.88[-1]$ & $9.01[1]$ & & \\
\hline 6 & $1.46[-1]$ & $7.89[1]$ & & & & & $3.49[-1]$ & $2.16[2]$ & & \\
\hline 7 & $3.97[-1]$ & $2.11[2]$ & & & & & & & & \\
\hline
\end{tabular}

${ }^{a}$ In units of $\mathrm{cm}^{3} \mathrm{~s}^{-1} \mathrm{~K}^{1.5}$.

${ }^{\mathrm{b}}$ In units of $\mathrm{eV}$. 
channel is better than $2 \%$ for $0.1 \leqslant T \leqslant 10000 \mathrm{eV}$. For the ${ }^{2} P_{3 / 2}$ state $2 s-2 p$ channel, the fit is better than $4 \%$ for 0.2 $\leqslant T \leqslant 10000 \mathrm{eV}$ and better than $14 \%$ for $0.1 \leqslant T<0.2 \mathrm{eV}$. The fit for the $2 p_{1 / 2}-2 p_{3 / 2}$ channel is better than $2 \%$ for 2 $\leqslant T \leqslant 10000 \mathrm{eV}$. Below $2 \mathrm{eV}$, the fit goes to zero faster than the calculated rate. However, at these energies, this channel contributes an insignificant amount to the total DR rate. For the $\Delta n \neq 0$ channels the fits are good to better than $1 \%$ for $160 \leqslant T \leqslant 10000 \mathrm{eV}$. Below $160 \mathrm{eV}$, the fits go to zero faster than the calculations. However, this is insignificant as these channels contribute less than $2 \%$ to the total DR rate for $T$ $<160 \mathrm{eV}$.

\section{SUMMARY}

We have calculated the total DR rate coefficients for ${ }^{2} P_{1 / 2}$ and ${ }^{2} P_{3 / 2}$ states in $\mathrm{Ti}^{17+}, \mathrm{Fe}^{21+}$, and $\mathrm{Mo}^{37+}$ ions including contributions from $2 s-2 p, 2 p_{1 / 2}-2 p_{3 / 2}, 2 \ell-3 \ell^{\prime}$, and $1 s-2 p$ excitations. These relativistic calculations are performed in intermediate coupling with a configuration interaction. We find that accurate Coster-Kronig energies are essential in the determination of reliable DR rate coefficients at low temperatures. We also find that the fine-structure excitation channel can be important at low temperatures for some ions.

\section{ACKNOWLEDGMENTS}

This work was performed under the auspices of the U.S. Department of Energy by Lawrence Livermore National Laboratory under Contract No. W-7405-ENG-48. D.W.S. was supported by NASA High Energy Astrophysics X-Ray Astronomy Research and Analysis Grant No. NAG5-5123.
[1] M. J. Seaton and P. J. Storey, in Atomic Processes and Applications, edited by P. G. Burke and B. L. Morseiwitsch (NorthHolland, Amsterdam, 1976), p. 133.

[2] A. Burgess, Astrophys. J. 139, 776 (1964); 141, 1589 (1965).

[3] J. Dubau and S. Volonte, Rep. Prog. Phys. 43, 199 (1980).

[4] H. P. Summer, Mon. Not. R. Astron. Soc. 169, 663 (1974).

[5] B. L. Whitten, A. U. Hazi, M. H. Chen, and P. L. Hagelstein, Phys. Rev. A 33, 2171 (1986).

[6] A. L. Merts, R. D. Cowan, and N. H. Magee, Jr., Los Alamos Scientific Laboratory Report No. LA-6220-MS, 1976 (unpublished).

[7] Recombination of Atomic Ions, Vol. 296 of NATO Advanced Study Institute, Series B: Physics, edited by W. G. Graham, W. Fritsch, Y. Hahn, and J. A. Tanis (Plenum, New York, 1992).

[8] H. H. Ramadan and Y. Hahn, Phys. Rev. A 39, 3350 (1989).

[9] I. Nasser and Y. Hahn, Phys. Rev. A 39, 401 (1989).

[10] N. R. Badnell, J. Phys. B 19, 3827 (1986).

[11] D. W. Savin, T. Bartsch, M. H. Chen, S. M. Kahn, D. A. Liedahl, J. Linkemann, A. Müller, S. Schippers, M. Schmitt, D. Schwalm, and A. Wolf, Astrophys. J. 489, L115 (1997).

[12] M. H. Chen, Phys. Rev. A 38, 2332 (1988).
[13] I. P. Grant, B. J. McKenzie, P. H. Norington, D. F. Mayers, and N. C. Pyper, Comput. Phys. Commun. 21, 207 (1980).

[14] M. H. Chen, Phys. Rev. A 31, 1449 (1985) .

[15] W. Bambynek, B. Crasemann, R. W. Fink, H. U. Freund, H. Mark, C. D. Swift, R. E. Price, and P. V. Rao, Rev. Mod. Phys. 44, 1458 (1974).

[16] I. P. Grant, J. Phys. B 7, 1458 (1974).

[17] J. Sugar and A. Musgrove, J. Phys. Chem. Ref. Data 19, 527 (1990); 22, 1213 (1993).

[18] A. Lampert, A. Wolf, D. Habs, J. Kenntner, G. Kilgus, D. Schwalm, M. S. Pindzola, and N. R. Badnell, Phys. Rev. A 53, 1413 (1996).

[19] D. T. Woods, J. M. Shull, and C. L. Sarazin, Astrophys. J. 249, 399 (1981).

[20] J. Wang, H. R. Griem, R. Hess, W. L. Rowan, and T. P. Kochanski, Phys. Rev. A 33, 4293 (1986).

[21] J. Wang, H. R. Griem, R. Hess, and W. L. Rowan, Phys. Rev. A 38, 4761 (1988).

[22] V. P. Zhdanov, Sov. J. Plasma Phys. 5, 3220 (1979).

[23] D. R. Inglis and E. Teller, Astrophys. J. 90, 439 (1939).

[24] M. Arnaud and J. Raymond, Astrophys. J. 398, 394 (1992). 